\title{
Téoros
}

Revue de recherche en tourisme

\section{Les modèles de demande en économie du marketing : une exploration interdisciplinaire}

\section{Pascal Tremblay}

Volume 7, numéro 3, novembre 1988

Économie du tourisme

URI : https://id.erudit.org/iderudit/1080384ar

DOI : https://doi.org/10.7202/1080384ar

Aller au sommaire du numéro

Éditeur(s)

Université du Québec à Montréal

ISSN

0712-8657 (imprimé)

1923-2705 (numérique)

Découvrir la revue

Citer cet article

Tremblay, P. (1988). Les modèles de demande en économie du marketing : une exploration interdisciplinaire. Téoros, 7(3), 40-44.

https://doi.org/10.7202/1080384ar d'utilisation que vous pouvez consulter en ligne. 


\section{Les modèles de demande en économie du marketing: une exploration interdisciplinaire}

\begin{abstract}
Introduction
La construction de modèles en tourisme a connu une certaine effervescence dans les dernières années et l'on a vu apparaître dans les revues spécialisées des analyses du tourisme relevant de champs $\mathrm{d}^{+}$intérêts divers. Tous les détracteurs du phénomène touristique ont souligné sa croissance, son importance pour les économies nationales et régionales ainsi que comme phénomène de consommation. Un des aspects ayant attiré I'attention de la majorité des chercheurs est celui de la demande tourisnique. Celle-ci est généralement définie comme la relation entre la quantité demandée de services touristiques et les facteurs qui l'influence.
\end{abstract}

Avee des objectifs différents, dans des contextes analytiques differents, specialistes de toutes disciplines se sont mis à étudier demandes et marchés et à essayer de leurs attacher des valeurs numériques; potentielles sources d'information pour la prise de décision. Il est intéressant de faire une brève exploration des méthodes qui semblent avoir bourgeonné de deux champs de spécialisation ayant été particulièrement prolifiques, l'économie et le marketing. Mon but n'est pas de me concentrer sur l'analyse technique des modèles mathematiques et statistiques utilisés dans les deux disciplines. Je m'intéresserai plutốt aux raisons d'ètre et aux implications des différents contextes théori= ques créant des divergences dans les deux approches. Je ferai des observations sur l'importance relative de certains types de modeles dans la littérature specialisée et traiterai des efforts de rapprochement récents.

Il va de soi que les différences majeures dans les méthodes utilisées dérivent de fossés théoriques entre l'économie et le marketing qui dépassent largement le cadre des modeles de demandes appliqués au tourisme. Pourtant, dans ces applications, les deux disciplines tentent souvent de répondre aux mêmes questions et semblent utiliser des méthodes quantitatives similaires. S'agit-il de différences majeures ou de détails n intéressant que les passionnés de méthodologie? Je risque de poser plus de questions que de donner de réponses. Mais le temps et le contexte semblent appropriés pour ce genre de réflexion. Le tourisme fut assez longtemps considéré par les chercheurs comme un potentiel terrain pour la coopération multidisciplinaire. La recherche en tourisme a maintenant existé assez longtemps pour qu'on puisse interroger les faits. Les mode- les de demande constituent un excellent terrain d'investigation.

\section{Modèles}

Le mot modéle a plusieurs interprétations. Dans le contexte scientifique, il est d'usage de traiter le sujet après avoir défini systèmes, théories, abstractions, hypothèses, paradigmes, structure et concepts. Pour éviter de $\mathrm{m}$ 'enliser dans la méthodologie scientifique (ce qui n'est sûrement pas le but du présent article), la définition la plus simple et générale peut servir. Un modèle doit être perçu comme une représentation simplifiée des relations essentielles entre les diverses parties d'une structure complexe. Cette définition devrait plaire a l'intuition et est assez générale pour ềtre acceptée par tous.

Une analyse des modeles de demande de tourisme qui se voudrait complète nécessiterait un travail multi-disciplinaire poussé. Mon but est de me limiter à une comparaison générale des approches utilisées en économie et en marketing parce qu'elles ont donné lieu à une quantité relativement importante de recherches reliées à la demande. Dans les deux cas, l'utilisation intensive de méthodes quantitatives (mathématiques et statistiques) pour mesurer la demande a amené une approche de type scientifique. Mais l"utilisation d'une telle approche ne constitue sutrement pas, en soi, une démonstration que l'economie ou le marketing sont des sciences ${ }^{(1 !}$. Le débat est d'une nature philosophique et est bien documenté. Pour l'éviter, je me contente de considerer l'économie et le marketing comme des disciplines. L'homogénéité relative quant aux buts, principes, objectifs et hypothèses de chaque discipline donne liew a des modèles relativement hétérogènes. Pour les besoins du présent article, on peut définir les frontières des disciplines par l'existence de communautés reconnues d'économistes et de marketers. Il y a, a l'intérieur de ces communautés beaucoup d'interactions mais peu d'échanges d'une communauté à l'autre. Cet état de chose est évidemment modifié quand des recherches multi-disciplinaires sont entreprises.

Les éléments communs aux modèles économiques et marketing existent avant tout au niveau de la procédure analytique générale. Celle-ci peut être résuméc par quelques étapes:

1- description d'hypothèses 
2- construction de la structure d"un modèle dérivant de 1

3- application de méthodes statistiques sur des données déjà existantes ou accumulées pour le modèle

4- résultats et conclusions sur la validité du modèle, particulièrement son potentiel pour la prédiction.

\section{Influences d'autres disciplines}

L'influence d'autres disciplines sur les modèles de marketing et d'économie se doit d'être reconnue et explique en elle-même certaines divergences entre les deux. Par exemple, l'apport de la psychologie, par l'entremise des theories du comportement, est un aspect fondamental de la théorie du comportement du consommateur en marketing. Les modèles de prise-de-décision du consommateur en sont directement dérivés et sont intégrés dans l'analyse de la demande en marketing.

Les sociologues et anthropologues ont un impact important sur les théories économiques, souvent en apportant des dimensions que les économistes peuvent difficilement inclure dans leurs modèles. Veblen a conçu une des critiques les plus sévères, et respectées, au sujet de la signification des modèles économiques servant à mesurer la demande et a démontré leur incapacité à incorporer d'importants déterminants de la demande pour des biens et services complexes dans un contexte d'abondance ${ }^{(2)}$. Il y soulignait, entre autres, l'importance du contexte institutionnel et de la culture pour des produits non-essentiels. Son ouvrage classique précédait l'analyse de McCannell sur l'importance des marqueurs et de la reconnaissance des attractions pour le tourisme $e^{(3)}$. Ces modèles qualitatifs très pertinents ne sont que très difficilement intégrés à la recherche économique. Mais le marketing y a puisé une part de son inspiration.

Les géographes ont, et continuent à avoir, une contribution importante au niveau de l'analyse spatiale des flux touristiques. Leur méthodologie a influencé l'étude marketing des réseaux de distribution. Ils sont aussi à l'origine des modèles de gravité utilisés par les économistes pour estimer la demande de voyages entre des destinations choisies, sur des circuits pré-établis ou pour des moyens de transport donnés.

Les méthodes statistiques uitlisées en économie et en marketing résultent de la sélection de méthodes répondant aux besoins propres des deux disciplines. Ces méthodes ont été adaptées et raffinées suffisamment pour étre associées étroitement à leur champs d'application; économétrie, psychométrie, sociométrie... Certaines techniques sont maintenant étroitement associées à des modèles de marketing telles que l'analyse conjointe, l'analyse multi-dimensionnelle (multidimensional scaling) et plusieurs autres. Ces modèles ne sont supposés servir qu"à falsi- fier des ênoncés théoriques et ne doivent pas constituer des fins en soi. Les méthodes de prévision font aussi parti du champ des statistiques et sont suffisamment développées et générales pour constituer une spécialisation en soi.

\section{Économie et marketing en tourisme}

J'ai déjà mentionné (et le lecteur l'aura déjà vérifié) que la présente analyse ne se veut pas rigoureusement scientifique, mais plutồt de type exploratoire. Mon intention n'est pas d'examiner en détail les différences méthodologiques des deux disciplines mais seulement de faire le point sur les directions qu'elles prennent dans la recherche appliquée en tourisme. Mon approche étant donc descriptive, il est utile que je mentionne les sources sur lesquelles repose mon analyse. Lors de précédents travaux sur les modèles de demande pour le tourisme international, j'ai parcouru une importante partie de cette littérature. J'ai aussi couvert une importante quantité de modèles marketing appliqués au tourisme lors d'un travail sur leurs utilisations potentielles dans un modèle économique. Je me base sur des observations dérivant d'articles ayant des origines assez diverses; soit de revues spécialisées en tourisme, en économie ou en marketing. principalement d'Europe et d'Amérique du Nord. J'ai cru intéressant de porter une attention particulière aux articles de deux revues spécialisées dans la recherche en tourisme; Annals of Tourism Research et Journal of Travel Research. Mon but était de survoler les introductions et conclusions des articles élaborant des modèles de demande pour essayer d'un extraire des généralisations. Un tel survol n'a qu'une valeur indicative car cet exercice n'est pas systématique et n'implique que deux revues américaines ayant des orientations propres. L'importance relative des différents modẻles qu'on y trouve ne reflète sürement pas le contenu de l'ensemble des revues spécialisées en tourisme. De plus, le traitement de ces modèles, provenant de milieux académiques, ne correspond sûrement pas à la fréquence de leurs usages dans le secteur privé.

Plusieurs ouvrages classiques sur la demande et la prévision en tourisme ont été consultés. J'ai cru intéressant de faire la comparaison entre le traitement de la demande dans les sections sur les aspects marketing et les aspects économiques au sein d'ouvrages généraux sur le tourisme, possiblement écrits par des généralistes. Le seul exercice de ce type que j'ai pu effectuer provient de deux publications de la collection Que sais-je?. Lanquar a participé à un ouvrage sur l'économie du tourisme ${ }^{(4)}$ et à un autre sur le marketing touristique ${ }^{(3)}$. Dans les premiers chapitres de chaque ouvrage, il traite de la demande touristique. Les différences entre les deux traitements peuvent servir de point de départ à la présente analyse.

Dans L'économie du tourisme, Lanquar débute son chapitre sur la demande touristique par une définition de la demande qui est en fait une définition nationale (macroéconomique) de la nature du tourisme acceptée par I"Organisation Mondiale du Tourisme. Il élabore ensuite une série de statistiques sur la répartition des flux touristiques, sur l'importance relative des demandes domestiques et extérieures et sur la saisonnalité. La section suivante traite des facteurs de la demande touristique et est typique des préoccupations des travaux économiques. Lanquar y établit une typologie des déterminants de la demande:

1 disponibilité de temps libre (institutions et marché du travail)

\section{2- facteurs économiques}

\section{3- facteurs démographiques}

\section{4- facteurs sociaux}

\section{5- organisation et promotion}

Ensuite, il définit la consommation touristique en termes de sa concentration dans le temps et l'espace (caractéristiques du tourisme) et en termes des industries traditionnelles qui la compose.

Dans Le marketing touristique, Lanquar et Hollier débutent la section sur la demande en soulignant l'importance pour toute organisation d'avoir un système d'information permettant la connaissance du "marché; c'est-à-dire de la demande" (cette définition du terme marché n'est sûrement pas universelle). Ils décrivent ensuite le concept de système d'informations (système opérationnel de recherche), son contenu et son évaluation. Ensuite, suivent des données sur les princpaux marchés, une section sur la segmentation des marchés, le röle des motivations et de la décision d'achat. Ils terminent avec un brève description des méthodes de recherche en marketing.

On apprend d'abord de cette comparaison que demande fait partie du vocabulaire economique et que marche du vocabulaire marketing. Les spécialistes de la recherche en marketing ${ }^{(6)}$ utilisent généralement des expressions un peu plus précises telles que le marché minimum, le marché potentiel et les prévisions de marché. Seule cette dernière peut être interprétée comme équivalente à la prévision de la demande. Mais l'utilisation du mot marché ou de marché potentiel en marketing suggère une attitude philosophique différenciant l'économie et le marketing. L'intérêt porté à l'existence d'un marché potentiel dont les dimensions sont déterminées par des facteurs socio-démographiques et dont les parts relatives conquises par les différents compétiteurs dépendent des interventions marketing (stratégies de promotion. produit, prix et position...) reflète la vision générale adoptée en marketing.

En économie, la demande est un concept 
théorique de base et qui est défini par la relation causale entre certaines variables explicatives (le plus souvent des variables typiquement économiques tels le prix des biens, le revenu, ...) et la variable expliquée; la quantité demandée du produit.

Les différences d'approche juste mentionnées ne sont ni incompatibles, ni exclusives. Elles ne font qu"introduire des perspectives qui donnent lieu à des approches et modèles avec des objectifs relativement proches, mais concentrant sur des aspects différents. Il en résulte des analyses spécialisées créant différents niveaux de cloisonnement intradisciplinaire. Un danger attaché à cet état de chose est que différentes méthodes abordant les mêmes problèmes généraux puissent être utilisées et interprétées par différents intervenants peu sensibilisés aux alternatives existantes pour arriver à des conclusions différentes. Les utilisateurs de ces résultats peuvent bien ne pas réaliser que les différences méthodologiques entre disciplines reflètent des orientations particulières qui ont des implications au niveau des décisions ou politique qu'elles suggèrent. L'importance de la recherche, des études de marché, études économiques, études d'impact, études coûtsbénefices sur lesquelles les entreprises, associations et gouvernements basent leurs décisions, rend essentielle une meilleure compréhension des bases philosophiques et méthodologiques de ces modèles par les généralistes en tourisme. S'il est possible de voir des tendances ou des biais dans les implications ou résultats de différents types d'études, le choix d'une approche pourrait facilement devenir le principal déterminant des conclusions d'une étude.

\section{Demande et économie du tourisme}

Les différents modèles de demandes économiques ont des caractéristiques propres plus ou moins rattachées aux courants dominants de la pensée économique (donc sủrement pas acceptés par tous). Les économistes construisent des modèles qui doivent idéalement avoir des bases thériques solidement intégrées à d'autres modèles acceptés et respectant leurs fondations théoriques. Généralement, les agents économiques sont rationnels et maximisent un index d'utilité pouvant inclure des biens de consommation, leurs revenus, leurs temps de loisirs ou d'autres variables appropriées. Les consommateurs typiquement maximisent l'utilité qu'ils dérivent de la consommation de certains biens et services et les producteurs maximisent les profits de leurs entreprises. Certaines hypothèses sont formulées sur les contraintes affectant leurs inter-relations, soit les structures de marchés, le degré d"incertitude, le niveau d'information, le cadre institutionnel et les cadres temporels et spatiaux où se situe l'interaction.

Les économistes parviennent à tester la validité de leurs modèles en imputant des valeurs numériques aux variables décrites et en quan- tifiant les fonctions d'utilité (ou des fonctions dérivées) et les contraintes sur le cadre économique. Pour ce faire, des méthodes statistiques (économétrie) établissant le degré de confiance devant ềtre associés à la validité de leurs modèles sont utilisées. Bien entendu, l'existence de données accessibles dans le format requis (séries chronologiques ou autres) pour un cadre d'analyse donné détermine en grande partie l'intérêt porté à la construction d'un modèle puisque sa validation sera possible. Les économistes vont, en général, utiliser des sources de données déjà existantes (données secondaires) et vont se contenter de manipuler et transformer ces dernières pour leurs besoins particuliers. Mais elles auront été préalablement assemblées par des organismes officiels ou des agences spécialisées dans l'obtention de banques de données. Ceci convient bien à la majorité des modèles économiques qui se veulent suffisamment généraux, et qui s'intéressent à des aggrégats. Il est possible de faire des comparaisons sur la validité des modèles quand les sources de données sont jugées indépendantes de la construction des modèles. Les économistes veulent établir des relations scientifiques indiscutables et ayant un certain degré d'universalité en testant leurs modèles avec des sources acceptées d'informations (données provenant de gouvernements) et des méthodes statistiques acceptées, reconnues par leur communauté. L'universalité des définitions, des sources de données, des méthodes de collecte par les agences officielles se doit d'éliminer les biais pouvant être rencontrés lors de l'utilisation de méthodes de recherches, paramètres, définitions, sources, instruments de mesure et échantillons douteux ou risquant de ne pas ètre acceptés par tous. Ceci est un problème reconnu par les économistes $\mathrm{s}^{4}$ intéressant au tourisme ${ }^{\text {7]! }}$.

En tourisme, comme dans les autres champs d'applications, les économistes s'adressent à des publics particuliers et ont des objectifs propres lorsqu'ils construisent des modèles. J'ai établi un sommaire des objectifs cités dans les trente-deux (32) articles sur des modèles de demandes économiques en tourisme que j'ai pu examiner. L'objectif dominant au sein de ces études est celui d'établir une mesure de la causalité (élasticités) entre la quantité demandée de services touristiques pour une région ou un pays et certaines variables comme le taux de change, le niveau des prix, le niveau de revenu des pays visiteurs et les coúts de transport. Ces objectifs ont des implications stratégiques pour les gouvernements qui veulent améliorer la situation de leur compte tourisme au sein de la balance des paiements. Le peu d'importance qui sem= ble être attaché à la prévision dans l'échantillon examiné est lié au fait qu'il existe des revues spécialisées en prévision, qui publient des articles sur les méthodes et résultats de leurs différents champs d'applications, incluant le tourisme.

Donc, les modèles économiques de demande touristique adressent des questions intéres- sant gouvernements et milieux académiques. Plusieurs types de modèles ont été historiquement développés pour des usages particuliers, pas nécessairement le tourisme. Par exemple, les premiers modèles de demande de services récréatifs dérivèrent des courbes de demande de type conventionnel, décrivant la relation entre le coût de transport et la quantité demandée pour ces services ${ }^{(8)}$. Ces modèles devaient servir à mesurer les surplus des consommateurs provenant de la consommation d'équipements récréatifs publics, utilisés dans les analyses coûts-bénefices effectuées lors de conflits concernant les usages alternatifs de lieux publics (exploitation forestière et minérale, conservation, récréation...).

Les modèles économiques spécialisés dans l'étude du transport (dérivant de modèles utilisés en géographie et en ingénierie) ont établi des relations entre les densités de population, les distances séparant les populations et la demande pour divers moyens de transport appelés modèles de gravité.

TABLEAU 1

Objectifs des modèles economiques en tourisme

\begin{tabular}{lcr}
\hline $\begin{array}{l}\text { Description de la demande } \\
\text { Mesure des elasticités }\end{array}$ & 2 & $(6 \%)$ \\
$\begin{array}{l}\text { Établir la demande pour des } \\
\text { stratégies de développement }\end{array}$ & 7 & $(58 \%)$ \\
Prédire la demande future & 4 & $(13 \%)$ \\
\cline { 2 - 3 } & 31 & \\
\hline
\end{tabular}

TABLEAU 2

Methodes quantitatives utilisees

\begin{tabular}{lcr}
\hline $\begin{array}{l}\text { Simple examen de séries } \\
\text { chronologiques }\end{array}$ & 4 & $(13 \%)$ \\
$\begin{array}{l}\text { Regressions } \\
\text { Analyses discriminantes et } \\
\text { combinaisons de méthodes }\end{array}$ & 42 & $(71 \%)$ \\
Analyse canonique & 1 & $(13 \%)$ \\
& 31 & \\
\hline
\end{tabular}

Les économistes spécialisés en commerce international ont modifié leurs modèles pour expliquer importations et exportations de services touristiques. Ces modeles sont devenus particulièrement populaires et ont atteint des niveaux de sophistication technique pouvant surprendre les généralistes en tourisme. Ills sont cependant demeurés très simples quand à leurs prémisses. Ils sont basés sur 1"hypothèse que les consommateurs de services touristiques font des choix résultant de prises de décisions rationnelles dans un contexte économique, avec les déterminants économiques traditionnels. Les développements techniques ont surtout visé à incorporer plus d'informations concernant l'environnement 
et les institutions affectant le tourisme et à incorporer de meilleures définitions des variables, par exemple en construisant de meilleurs index des changements dans les coúts de transport. Ce type de modéle est surtout utilisé pour déterminer l'inpact de changements des taux de change ou des coûts de uransport sur les recettes nationales provenant du tourisme.

Les méthodes quantitatives utilisées reflètent I'importance attachée à la régression multiple en économétrie. Cette dernière est directement liée aux besoins dérivant de l'estimation des relations entre variables explicatives et variables expliquées. La complexité grandissante des modèles est parallèle à l'évolution des techniques statistiques et aux améliorations dans la qualité de données permettant de nouveaux raffinements. Mais ces applications de plus en plus spécialisées forcent les généralistes à acquérir une compréhension plus poussée des méthodes quantitatives, sans qu'ils ou elles possèdent nécessairement un bagage suffisant sur les limites et défauts des modèles de base.

\section{Demande et marketing touristique}

Les modèles marketing d'estimation de la demande touristique montrent moins d'uniformité dans leurs objectifs, leurs hypothèses et l'utilisation de méthodes quantitatives que les modèles économiques. Il n'est pas aisé d'essayer de les catégoriser par objectifs ou même par méthodes quantitatives car il est commun de retrouver une multiplicité des deux éléments au sein d'une recherche ou d'un article.

L'analyse-type étudiant la demande pour un produit, forfait ou destination vise le plus souvent à segmenter le marché pour permettre d'établir une stratégie marketing basée sur les caractéristiques des segments intéressant pour la prise de décision. Un autre objectif retrouvé assez fréquemment dans les modèles de tourisme est l'établissement d"un index d'attraction pour des produits touristiques. Il s'agit par exemple d'établir la position concurrentielle de destinations compétitionnan pour l'obtention de ventes à certains seg. ments de marchés. Ces analyses émettent l'hypothèse que le degré d'attraction dépend des caractéristiques de la population visitée et des caractéristiques du produit, de la distance séparant ce produit des marchés potentiels et des caractéristiques (préférences, attitudes, ...) des segments de marché constituant les marchés potentiels. Ce type d'analyse est étroitement lié à la méthodologie de segmentation de marchés.

La segmentation socio-démographique étant jugée insuffisante ou moins pertinente pour les marchés touristiques que la segmentation par attitudes ou styles-de-vie (lifestyles), plusieurs modèles se sont concentrés sur l'établissement de relations entre la demande de services touristiques et les caractéristiques psychographiques (psychographics) de divers segments. L’approche typique débute par l'identification de facteurs importants dans la détermination de la demande (factor analysis). Ces facteurs sont ensuite utilisés pour catégoriser les segments ayant des caractéristiques propres avec des méthodes statistiques appropriées (cluster analysis ou discriminant analysis). Pour conclure, on essaie d'établir l'existence de relations intéressantes et différenciées entre caractéristiques retenues pour catégoriser les segments et les comportements des consommateurs constituant ces segments à l'aide de méthodes de régression.

Une multitude d'instruments quantitatifs aidant à exécuter ces opérations existe, les plus communs ayant été mentionnés. D'autres, comme l'analyse multi-dimensionnelle (multi-dimensional scaling), ne sont que des manipulations permettant la transformation de données numériques en représentations géométriques. On peut, par exemple, représenter à l'aide de graphiques à deux ou trois dimensions, les perceptions d'un échantillon de population vis-ä-vis la relation entre des produits ou destinations et deux ou trois dimensions jugées importantes comme déterminants de la demande pour ces produits ou destinations concurrentiels.

L'analyse théorique proprement dite se situe dans l'établissement des caractéristiques à être utilisćes comme déterminants potentiels de la demande, la définition des critères de mesure, l'établissement de la relation fonctionnelle entre attitude, perception, degre d'attraction et comportement. Les économistes gardent généralement leurs distances par rapport à ces modèles à cause du côté à prior des étapes mentionnées ci-haut. Les variábles et leurs mesures sont définies selon les besoins particuliers de chaque étude et reposent rarement sur des théories reconnues par la communauté du markering. Leur caractère exploratoire et arbitraire empêche la comparaison des résultats entre études ou entre auteurs, la méthodologie étant rarement uniforme.

En fait, ces modèles avec leurs structures propres n'essaient pas d'établir des relations naturelles et universelles entre variables explicatives et variables expliquées. Ils ne servent qu'à définir des dimensions utiles. pour la prise de décision regardant les stratégies de marketing d'entreprises, associations, industries et gouvernements. L'étude des relations entre les produits et leurs marchés ne servent qu'à orienter les intéressés dans leurs actions marketing: pour leur permettre de transformer pouvoir d'achat en demande effective.

En marketing, les moyens stratégiques permettant d'affecter la demande à la disposition des offreurs jouent le rôle central dans l'analyse. Le focus qui est mis sur leur importance crée le besoin de définir des instruments pratiques et différenciés (définitions, critères, mesures...) avec des formats propres à chaque contexte. Il en résulte que des données primaires doivent généralement être assemblées et que leur obtention constitue, en soi, une partie importante de la recherche.

\section{Convergences ou divergences}

Tout généraliste en tourisme, chercheur ou intervenant, aura un jour ou l'autre à discuter et peut-étre quantifier la demande pour certains produits touristiques. Pour ce faire, des méthodes et instruments appropriés au contexte de l'étude seront généralement utilisées. Les clients, avec leurs besoins parti= culiers feront sưrement appel à la bonne catégorie de spécialistes. L'administration hôtelière désirant connaitre les marchés prospectifs ou potentiels pour un produit woudra être informée sur la répartition des clients potentiels par régions, catégories professionnelles, préférences de loisirs, styles de voyages, revenus discrétionnaires... La commission en charge de déterminer l'impact d'éventuelles taxes hôtelières utilisera un modèle économique où la définition du prix des services hôteliers et l'évaluation de l'élasticité-prix de la demande pour ces services domineront l'analyse. Sachant que les demandeurs de ces modeles vont probablement utiliser ce qui est convenable à leurs besoins, pourquoi vouloir que les généralistes soient en mesure de différencier les approches et leurs conséquences? Pourquoi les spécialistes pourraient-ils avoir un intérét à transcender les frontières reconnues de leurs disciplines?

Le développement même de recherches multidisciplinaires est la source du questionnement à la base du présent article. Des malaises qui ont longtemps existé quand les individus se mêlent de comparer des approches entre disciplines, qui posent des questions relativement similaires, se doivent de resurgir lorsqu'un sujet d'étude ou un champ d'intérết implique quelque forme de multidisciplinarité que ce soit. Les comparaisons méthodologiques sont vouées à mobiliser une grande partie de l'énergie dépensée dans ce type d'exercice, expliquant en partie leur rareté, en tourisme comme ailleurs.

Déjà, dans un souci de catégorisation des approches, Stafford a défini des paradigmes propres à la recherche en tourisme ${ }^{(9)}$. Ma réflexion ne veut pas débattre la place de l'économie, du marketing et du tourisme dans les sciences sociales. Je ne fais que constater que des disciplines bien établies, qui ont développé des techniques analytiques reconnues, sont utilisées pour répondre à des questions générales et d'intérêt commun touchant le tourisme et peuvent amener à des conclusions et perspectives suffisamment éloignées pour empécher leur comparaison ou complémentarité. L'attitude des chercheurs a été, sauf exceptions, d'éviter ces terrains dangereux où la pluparr, incluant le présent auteur, n'ont pas l'expertise qui pourrait permettre une comparaison poussée entre disciplines parentes.

Dans les revues spécialisées comme Annals of Tourism Research, Journal of Travel 
Research, Téoros et Tourism Management, on tente de réunir les avis de chercheurs de plusieurs disciplines. Les articles sont jugés par des experts de chaque discipline. On y retrouve une multitude $\mathrm{d}^{+}$articles touchant le tourisme qui se recoupent très peu à l'extérieur des disciplines. Il est facile, et probablement réaliste, d'imaginer que les lecteurs de ces revues portent leur intérêtt sur la discipline qui les intéresse et délaissent les autres. Pourtant, en tourisme, plus que partout ailleurs, la complexité du sujet et l'incapacité des modèles traditionnels suggèrent d"aller puiser de nouvelles ressources dans les autres disciplines.

On peut emettre diverses hypothèses quant aux directions qui seront prises par la recherche en examinant le cas des modeles de demande. L'intérềt porté au tourisme grandissant, on pourrait tout d'abord envisager que chaque discipline créera un grand nombre de chercheurs, qui à l'intérieur de leurs disciplines voudraient appliquer leurs travaux au tourisme. Cette profusion pourrait conduire à l'élargissement du fossé entre les disciplines par l'adhérence des revues spécialisées a un nombre limité, dans le pire cas à une seule, de disciplines ou facettes du tourisme par revue. Dejä, on peut observer qu'il existe une tendance pour ATR à publier des modèles économiques et pour JTR à dominer en marketing. Cette spécialisation informelle connaît bien des exceptions, mais pourrait dans le futur ajouter aux dejà nombreuses barrières institutionnelles empechant une collaboration interdisciplinaire plus poussée que l'existante juxtaposition d'articles spécialisés dans les revues.

Au contraire, peut-etre que les chercheurs en tourisme auront une différente attitude. Il est possible que les limites du bagage méthodologique existant dans chaque discipline créent la motivation nécessaire à développer des qualités de généralistes qui seraient profitables. Mais trop souvent, on associe généralistes et manque d'expertise. Espérons qu'il sera possible de voir des généralistes ayant quelques spécialites.

Déjà, des convergences existent et des modèles tentent de combiner les apports du marketing et de l'économie. Par exemple, les modeles économiques de tourisme international ont tendance à incorporer plus de variables marketing et autres (telles que les budgets marketing, les événements spéciaux, les changements institutionnels, les événements politiques comme le terrorisme... ${ }^{(10 i}$ ) afin de mieux expliquer les changements dans la demande. Mais on est toujours loin de l'inclusion, faite en marketing, de variables touchant directement aux attitudes, perceptions, préférences...

L'utilisation par des économistes de l'approche par caractéristiques (characteristic approach ${ }^{(11)}$ ) constitue aussi un pas dans la direction d'un compromis. Cette approche est basée sur 1'hypothèse que les consommateurs ne demandent pas des biens ou servi- ces comme tel, mais plutòt qu'ils demandent les attributs ou qualités incorporés dans ces biens et services. On peut imaginer que la demande pour un établissement hôtelier soit une fonction de la demande pour les differents services rendus par l'établissement. Il serait donc plus significatif de comparer les demandes pour ce genre de produits en incluant les caractéristiques de ces produits. Cette perspective se rapproche de certains modèles de marketing, tel que les modèles de décision d'achat basés sur les caractéristiques des produits. Elle n'est pas restrictive au niveau de la définition des biens et services car elle permet d'intégrer les changements dans les produits. Il a toujours été problématique pour les économistes d'établir des relations entre le prix des produits et la quantité qui en est demandée quand les qualités des produits varient dans le temps. En tourisme, les produits (forfaits, resorts, destinations, ....) possèdent des composantes qui changent beaucoup dans le temps. Les changements dans le produit ont toujours constitué une préoccupation centrale de la stratégie marketing, délaissée par les économistes. Un intéressant exemple d'application de ce genre de modèle a été réalisé par Morey $y^{(12)}$. La demande pour le ski alpin au Colorado est estimée et les caractéristiques des centres de ski (leurs attributs) y jouent un rôle important.

En marketing, l'importance grandissante des modèles à attributs multiples (multi-attribute models) et de l'analyse conjointe pour évaluer les préférences ne peut que reféter des rapprochements entre les intéréts et préoccupations des deux disciplines. Les deux techniques servent à la construction de fonctions d'utilité qui peuvent posséder la même structure que celles impliquées par les économistes dans leurs modeles. Il y a des similarités structurelles importantes entre les modeles à atuributs multiples et l'approche des caractéristiques. La différence fondamentale est que dans cette dernière, les économistes insistent pour utiliser des attributs physiques (ex.: longueur des pentes de ski. précipitations annuelles, ...) et qu'en marketing les attributs sont souvent subjectifs. Ils relèvent le plus souvent de la perception des consommateurs (qualités scéniques. réputation du centre de ski, atmosphère, ....).

Tous ces exemples démontrent que des rapprochements techniques sont envisageables. Cependant, les différences philosophiques et méthodologiques demeurent. Les économistes ne sont pas prêts à accepter que des caractéristiques évaluées subjectivement et mesurées par des instruments douteux puissent servir de base à des théories scientifiques. De même, les chercheurs en marketing ne sont pas près à se limiter aux descriptions $5 \mathrm{im}$ plistes du consommateur rationnel que les économistes utilisent et qui limiteraient beaucoup le devveloppement de stratégies de marketing. Espérons seulement que la discussion soit ouverte. $f$
Notes explicatives

(1) HUNT, Shelby D., Marketing Theory-The Philosophy of Marketing Science, Homewood, 1linois, 1943.

(2) VEBLEN, Thorstein. The Theory of the Leisure Class. New York, 1899.

(3) MACCANNELL, D., The Tourist: A New Theory of the Leisure Class, New York, 1976.

(4) LANOUAR, Robert, L'ecenomie du tourisme. Que sais-je?, Paris, 1963.

(5) LANQUAR, Robert, HOLLIER, Robert, Le marketing touristique. Que sais-ie?, Paris, 1981.

(6) KINNEAR, Thomas C., TAYLOR, James R., Marketing Resaarch. Singapore, 1987.

(7) WHITE, Jenneth J., WALKER, Mary Beth, Trous. ble in the Travel Account, Annals of Tourism Research, Vol. 9, 1982, pp. 37-56.

(8) CLAWSON, M., KNETSH, J.L, Economics of Outdoor Recreation, Ressources for the Future, 1986.

19) STAFFORD, J., Les paradigmes de la rechenche on téorologie: eitude, analyse et critique, Leisirs et Societes. Vol. 8, No 2, automne 1985, pp. 5.9-560.

(10) WIT, Stephen F., MARTIN, Christine A., Inrernational Tourism Demand Models-inclusion of Marketing Variables. Tourism Management. March 1987, pp. 33-40.

(11) LANCASTER, Kelvin, Consumer Demand: A New Approach, Columbia University Press, 1971. DOMENCICH, T.A., MCFADDEN, D., Urben Travel Demand: A Behavioral Analysis. North. Holland, 1975.

(12) MOREY, E.R.. The Demand for Site-Specific Recreational Activities: $A$ Chavacreristic $A \bar{p}$. prosch. Journal of Environmental Economics and Management, No 9, 1991, pp. 345-371. 